\title{
ANÁLISE DA INCERTEZA DO MAPEAMENTO BATIMÉTRICO DO CANAL DE NAVEGAÇÃO DO PORTO DO MUCURIPE (CE) - BRASIL
}

\author{
uncertainty analysis of bathymetric mapping of the navigation \\ Channel in Mucuripe harbor (CE) - Brazil \\ Eduardo Guilherme Gentil de Farias* \\ Luís Parente Maia**
}

\begin{abstract}
Resumo
A atividade portuária representa uma importante fonte de geração de renda e divisas, sendo considerada como a chave para o desenvolvimento sócio-econômico regional. Assim, faz-se necessário o constante monitoramento dos canais de navegação, uma vez que a boa manutenção das cotas batimétricas, contribui diretamente para a segurança no tráfego de embarcações. Para isso, são normalmente realizadas dragagens em áreas adjacentes às instalações portuárias. Nesse âmbito, o levantamento batimétrico é a metodologia mais adequada para o monitoramento dos canais de navegação após a dragagem. Apesar de sua relevância, há pouca discussão sobre a análise da precisão dos mapas batimétricos obtidos. Neste contexto, o avanco recente das técnicas computacionais aplicadas a navegação costeira, pode facilitar a verificação de possíveis incertezas no mapeamento. Assim, este estudo buscou contribuir para a melhoria das técnicas de análise batimétrica com base na geração de um mapa de incerteza através de análise geoestatística. Os resultados demonstram a viabilidade e conveniência de implementação de técnicas de krigagem por indicação, permitindo a análise da incerteza decorrente da interpolação comum (krigagem universal)
\end{abstract}

Palavras-chave: Navegação, Geoprocessamento, Gestão Portuária.

\begin{abstract}
The harbor activity represents an important source of income generation and foreign exchange is regarded as the key to regional socio-economic development. So, it is necessary the constant monitoring of the navigation channels, since the good state of repair, directly contributes to safety in boat traffic. For this, are usually carried out dredging in areas adjacent to port facilities, and the bathymetric survey the most appropriate methodology for monitoring the depths after the dredging. However, there is little discussion regarding the analysis of the accuracy of the bathymetry maps. In this context, the recent advancement of computational techniques applied, can facilitate the achievement of a detailed study to ascertain potential areas of uncertainty in a survey previously conducted. Thus, this study sought to contribute to the improvement of bathymetry techniques based on geostatistics from the generation of a map of uncertainty, seeking review of the accuracy of surveys in harbor zones. The results show the feasibility and advisability of implementing techniques of indicator kriging by allowing the analysis of the uncertainties arising from the ordinary interpolation.
\end{abstract}

Key words: Navigation, Geoprocessing, Port Management.

\section{Résumé}

L'activité portuaire représente une importante source de génération de revenus et de devises, étant considéré comme la clé de développement socio-économique. Ainsi, il est nécessaire fair la surveillance constante des chenaux de navigation, depuis le maintien de bonnes profondeur bathymétriques, contribue directement avec la sécurité du trafic maritime. Pour ce faire, le dragage est généralement effectuée dans les zones aux installations portuaires. Dans ce contexte, la bathymétrie c'est le méthode plus appropriée pour la surveillance des chenaux de navigation après le dragage. Malgré sa pertinence, il n'ya pas études de l'analyse de l'exactitude des cartes bathymétriques obtenues. Dans ce contexte, le développement récent de techniques de calcul appliquées à la navigation, peut faciliter la vérification des incertitudes possibles dans la cartographie. Ainsi, cette étude a cherché à contribuer à l'amélioration des techniques d'analyse basées sur la génération d'une carte bathymétrique de l'incertitude par l'analyse géostatistique. Les résultats démontrent la faisabilité et l'opportunité de la mise en œuvre des techniques de krigeage indication, permettant l'analyse de l'incertitude due à l'interpolation commune (krigeage universel).

Mots clés: Navigation, SIG, Gestion Portuaire.

(*) Doutorando no Programa de Pós-Graduação em Sensoriamento Remoto do Instituto Nacional de Pesquisas Espaciais - Cx. Postal 515, CEP: 12227-010, São José dos Campos (SP), Brasil, Tel.: (+ 55 12) 31868525 - gentil@dsr.inpe.br

(**) Bolsista Produtividade do CNPq, Prof. Dr. do Instituto de Ciências do Mar da Universidade Federal do Ceará - Cx. Postal 6025, CEP: 60165-081, Fortaleza (CE), Brasil, Tel.: (+55 85) 33667001 - parente@labomar.ufc.br 


\section{INTRODUÇÃO}

Com um litoral de $8500 \mathrm{~km}$ navegáveis, o Brasil possui um setor portuário que movimenta anualmente cerca de 700 milhões de toneladas das mais diversas mercadorias, respondendo sozinho, por mais de $90 \%$ das exportações (SEP, 2010). Desse modo, a atividade portuária representa uma importante fonte de geração de renda e divisas, atuando como importante veículo de promoção para o desenvolvimento socioeconômico regional (CLARK, 1996).

Buscando aliar sustentabilidade e segurança, o Brasil desde 25 de maio de 1980 por força do Decreto Legislativo no 11/80 é regido pela Convenção Safety of Life at Sea-SOLAS e assumiu o compromisso de implementar através deste código o International Ship and Port Facility Security-ISPS, que entrou em vigor em julho de 2004 (IMO, 2010).

O código ISPS, foi elaborado pela Organização Marítima Internacional (IMO), pertencente à Organização das Nações Unidas (ONU) e visa o estabelecimento de regras que tornem os navios e instalações portuárias mais seguras, considerando como relevantes a delimitação do perímetro portuário, adoção de instalações de segurança e monitoramento de estruturas e vias aquaviárias de acesso aos terminais portuários (IMO, 2010).

No tocante a preservação dos acessos náuticos aos terminais portuários, a dragagem de manutenção representa atualmente a solução mais amplamente difundida e adotada na preservação das profundidades do canal de navegação, bacias de evolução e berços de atracação, em zonas cuja lâmina d'água estiver sendo progressivamente reduzida, devido ao assoreamento (FLEMER et al., 1997).

Dentre as ações que visam à formulação de planos de dragagem, o mapeamento batimétrico é de grande importância para estudos preliminares e monitoramento dos trabalhos portuários. Esta técnica pode ser definida como a análise do reconhecimento da morfologia do substrato de um corpo d água por intermédio de medições da profundidade local. Para isso, é utilizado o equipamento denominado ecobatímetro que mede a profundidade através de transmissão e recepção de ondas sonoras (FILHO, 2004).

Nesse íntere, a adoção de métodos de análise espacial, em particular de técnicas de krigagem por indicação, podem identificar as regiões de maior incerteza no levantamento, ressaltando locais aonde o grau de amostragem é insuficiente, definindo assim, o zoneamento da precisão do mapeamento obtido.

Partindo dessa premissa, o presente trabalho visa utilizar técnicas de krigagem por indicação nos dados obtidos por ecobatimetria da região do porto do Mucuripe, localizado na cidade de Fortaleza, nordeste do Brasil.

\section{MATERIAL E MÉTODOS}

O canal de navegação do porto do Mucuripe encontra-se situado no município de Fortaleza, região Nordeste do Brasil, sob as longitudes -37o29'00"W e -37o28'00"W e entre as latitudes -3o43'00"S e -3o41'30"S (Figura 1), possuindo uma posição geográfica estratégica na costa brasileira, por ser um dos grandes portos mais próximos da América do Norte e Europa, fator importante na redução do custo do transporte. 


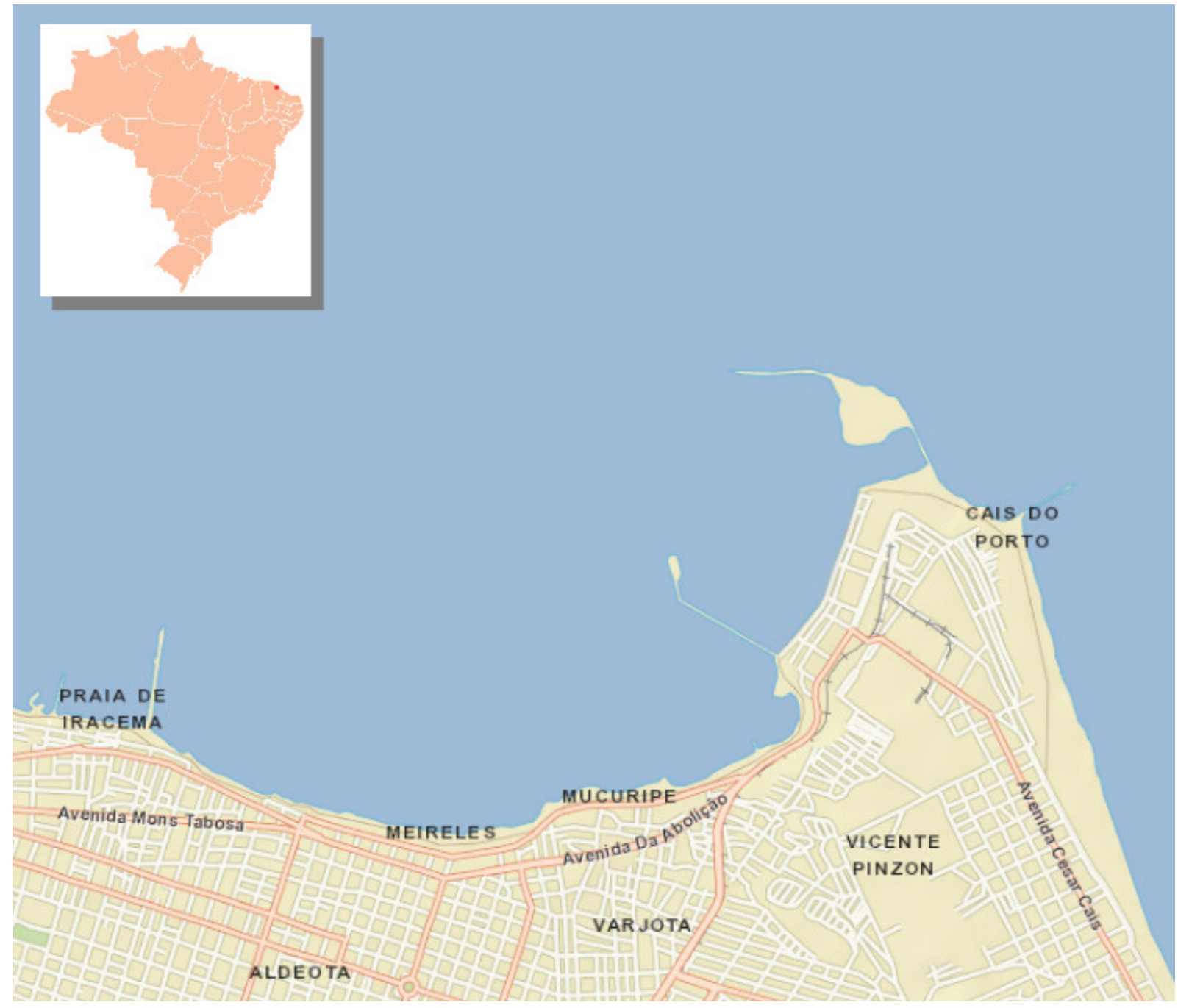

Figura 1 - Região costeira de Fortaleza.

A região do cais do porto do Mucuripe encontra-se destacada no canto direito do mapa.

\section{Levantamento Batimétrico}

O mapeamento de campo, ocorreu no dia 26 de Outubro de 2007, durante o período de preamar de sizígia. Para este trabalho, foi utilizada uma ecossonda conjugada a um GPS, modelo GPSMAP 238 Sounder GARMIN, transdutor de resolução de 0,01metros de profundidade, sensor de temperatura, antena externa com recepção para 12 canais e receptor diferencial. A ecossonda implementa um sistema de aquisição de dados de profundidade, hora e coordenadas, acoplado a um laptop. A taxa de transmissão dos dados é de 1/seg sendo capturados no formato NMEA na versão 2.3.

O levantamento batimétrico foi realizado a bordo do barco de pesquisa Professor Martins Filho da Universidade Federal do Ceará. Durante as atividades, foram realizados transectos regulares com espaçamento de 100 metros em todo o canal de navegação do porto.

Os dados foram salvos em um laptop em formato ASCII no padrão NMEA 2.3. As sentenças utilizadas para o processamento foram a GPGLL e SDDBT onde trazem as informações para elaboração de tabelas no formato XYZ, coordenadas e profundidades.

Os valores de profundidade foram corrigidos ao nível reduzido da Diretoria de Hidrografia e Navegação - Marinha do Brasil (DHN) com o objetivo de eliminar o efeito da maré, utilizando como base a tábua de maré do porto de Fortaleza, objetivando dar suporte a correta modelagem do mapa batimétrico. 


\section{Krigagem.}

A krigagem é representada por um conjunto de técnicas de regressão linear generalizadas, visando minimizar uma variância de estimação a partir de um modelo de covariância definido a priori (Deutsch e Journel 1998).

Consiste num processo de estimativa por médias móveis, de valores de variáveis distribuídas no espaço a partir de valores adjacentes, considerados como interdependentes, estimando o valor de um atributo, em uma posição u não amostrada, a partir de um conjunto de amostras vizinhas $\mathrm{Z}(\mathrm{u} \alpha), \alpha=1, \ldots, \mathrm{n}$. Desse modo, possibilita inferências de valores, a partir de amostras pontuais de um atributo espacial.

Supondo-se a existência de um conjunto de $n$ amostras pontuais, $Z(u \alpha)$, de valores de um atributo, dentro de uma região A da superfície terrestre, temos segundo a equação 1, a seguinte condição:

$$
Z\left(u_{\alpha}\right), u_{\alpha} \in A \subset R 2 e \alpha=1,2, \ldots, n
$$

O procedimento de krigagem permite a estimação de valores do atributo em localizações u $\neq u \alpha$. Além disso, este procedimento possibilita a inferência da variância de krigagem, que, em certas condições, que serão discutidas neste trabalho, podem ser utilizadas como uma medida de incerteza do atributo em $u$.

A krigagem reúne diversos tipos de métodos de estimação, como: krigagem simples, ordinária, universal, por indicação, disjunta e cokrigagem. Porém, este estudo só tratará da krigagem por indicação.

\section{Krigagem por indicação}

A krigagem por indicação é um estimador de krigagem não linear, ou seja, um estimador de krigagem linear aplicado a um conjunto de dados que foram modificados segundo uma transformação gaussiana, lognormal ou outra (Deutsch e Journel, 1998). Na krigagem por indicação, é necessária a realização de uma transformação não linear sobre o conjunto de dados amostrais $Z(u \alpha)$, a qual é denominada codificação por indicação.

A principal vantagem deste método, é a de ser uma técnica não paramétrica, ou seja, nenhum tipo de distribuição para a variável aleatória (VA) é considerado a priori, o que possibilita uma estimativa da função de distribuição para a VA, permitindo a determinação de incertezas e a inferência de valores do atributo em locais não amostrados (Felgueiras, 1999).

$\mathrm{Na}$ krigagem por indicação, utiliza-se função distribuição acumulada, a fdac, $\mathrm{F}(\mathrm{u} ; \mathrm{z} \mid(\mathrm{n}))$, visando modelar a incerteza sobre os valores de $\mathrm{Z}(\mathrm{u})$, em posições u não amostradas, permitindo uma aproximação da fdac.

Como já foi dito anteriormente, a krigagem por indicação requer que os valores dos atributos sejam modificados segundo uma transformação não linear, a codificação por indicação. Um conjunto amostral por indicação $\mathrm{I}(\mathrm{u}=\mathrm{u} \alpha)$ para um valor de corte $\mathrm{zk}$ é gerado através da codificação por indicação aplicada a um conjunto de dados $Z(u=u \alpha)$ segundo a equação 2 :

$$
I\left(u ; z_{k}\right)=\left(\begin{array}{l}
1, i f Z(u) \leq z_{k} \\
0, i f Z(u)>z_{k}
\end{array}\right)
$$

O modelo de variografia é obtido através do conjunto amostral codificado com valores de 0 e 1, o qual depende da existência mínima de 0 's e 1 's no conjunto de amostra. O conjunto amostral codificado é utilizado para inferir valores para variáveis aleatórias por indicacão I(u; zk), com $u \neq u \alpha$. 
Segundo Felgueiras (1999), a krigagem por indicação é dada pela equação 3:

$$
F_{s}^{\Psi^{\prime}}=\left(u ; z_{k} /(n)\right)=\sum_{\alpha=1}^{n(u)} w_{\alpha}\left(u ; z_{k}\right) ;\left(u_{\alpha} ; z_{k}\right)+\left[1-\sum_{\alpha=1}^{n(u)} w_{\alpha}\left(u ; z_{k}\right) F^{\Psi^{\prime}}\left(z_{k}\right)\right]
$$

Onde:

$\mathrm{F} \Psi(\mathrm{zk})$ é a média da função aleatória da região estacionária; estimação.

$\mathrm{S} \alpha(\mathrm{u} ; \mathrm{zk})$ são os pesos determinados com o objetivo de minimizar a variância do erro da

De acordo com Felgueiras (1999), a krigagem por indicação, fornece estimativas para cada valor de corte $\mathrm{zk}$, que é também a melhor estimativa mínima quadrática da esperança condicional da VA I(u, zk). Calculando as estimativas para diversos valores de corte da fdac de Z(u), tem-se uma aproximação discretizada da fdac real de Z(u). Por isso, quanto maior for o número de cortes melhor será a aproximação.

\section{Análise por semivariograma.}

O semivariograma permite representar quantitativamente a variação de um fenômeno regionalizado no espaço, constituindo-se como uma ferramenta básica de suporte as técnicas de krigagem (Alcântara, 2006). Segundo Barbosa (2005), os parâmetros de análise por semivariograma (Figura 2), são os seguintes:

. Alcance: distância dentro da qual existe correlação espacial entre as amostras;

. Patamar: é o valor que exprime o alcance do semivariograma. Os valores amostrais que se encontram dentro do intervalo de patamar apresentam dependência espacial;

. Efeito pepita: Revela a descontinuidade do semivariograma para distâncias inferiores do quea menor distância amostral. Idealmente este valor deverá tender para zero.

. Contribuição: é expresso como sendo a diferença entre o patamar e o efeito pepita.

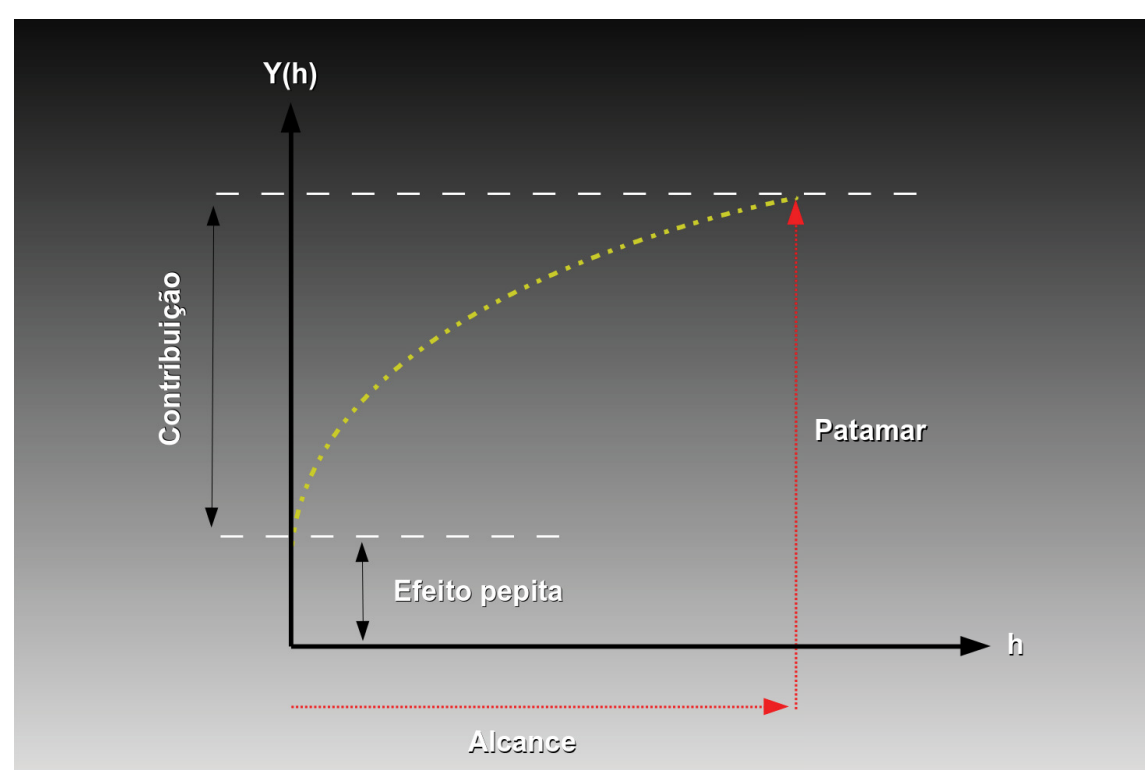

Figura 2 - Modelo de semivariograma (Adaptado de Barbosa, 2005). 


\section{RESULTADOS E DISCUSSÃO}

Os dados batimétricos são normalmente coletados a partir de amostras pontuais (Li et al., 2011). Desse modo, a estimativa de valores para locais desconhecidos a partir de técnicas de interpolação, torna-se uma ferramenta essencial para a geração de superfícies espacialmente contínuas (Verfaillie et al., 2006). Nesse contexto, a estimativa da precisão dos modelos digitais obtidos devem ser avaliados, uma vez que, em atividades ligadas a navegação, erros significativos de cotas batimétricas podem causar prejuízos as embarcações, em decorrência de eventuais encalhes.

Para nossa região de estudo, foram avaliados 5030 pontos de batimetria referentes ao canal de navegação do porto do Mucuripe - CE. Numa análise exploratória inicial, é possível evidenciar que a profundidade média para esta região, é de -10.11 metros, com presença de picos máximos da ordem de aproximadamente - 14 metros e desvio padrão de 2.75 metros. Seguindo a metodologia descrita por Feigueiras (1999), foram adotados como valores de corte, as variáveis referentes ao quartil inferior, mediana e quartil superior, tendo estes apresentados os valores de $-11.85,-10.92 \mathrm{e}$ -8.75 respectivamente.

A geração dos modelos de semivariograma, analisou nosso conjunto de dados, a partir de uma distribuição unidirecional com amostragem irregular. O modelo transitivo esférico, foi adotado para ajuste gráfico, uma vez que este, apresentou melhor aderência a distribuição das linhas de corte analisadas (Figuras 3, 4 e 5).

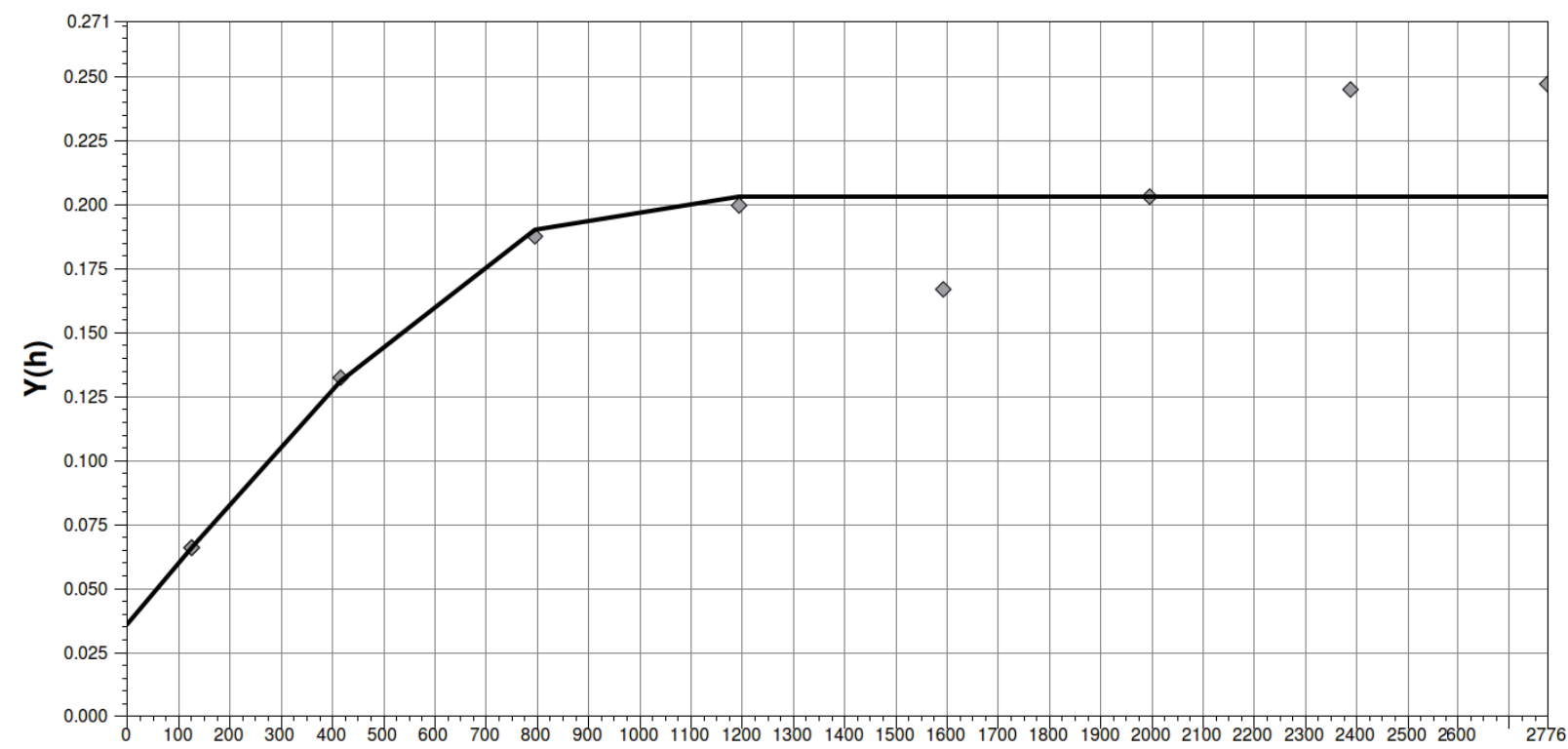

Distância

Figura 3 - Ajuste de semivariograma para o quartil inferior.

Foi possível observar baixos valores de efeito pepita para os três limiares de corte, sendo estesda ordem de 0.036 para quartil inferior, 0.047 para mediana e 0.025 para quartil superior, o que mostra uma baixa descontinuidade para distâncias menores que a menor distância entre as amostras, sugerindo uma variabilidade espacial de pequena escala para nossa região de estudo.

Os valores de alcance encontrados para quartil inferior, mediana e quartil superior foram 1387, 1387 e 1361 respectivamente, o que demonstra uma alta correlação espacial entre o conjunto de dados analisados. 


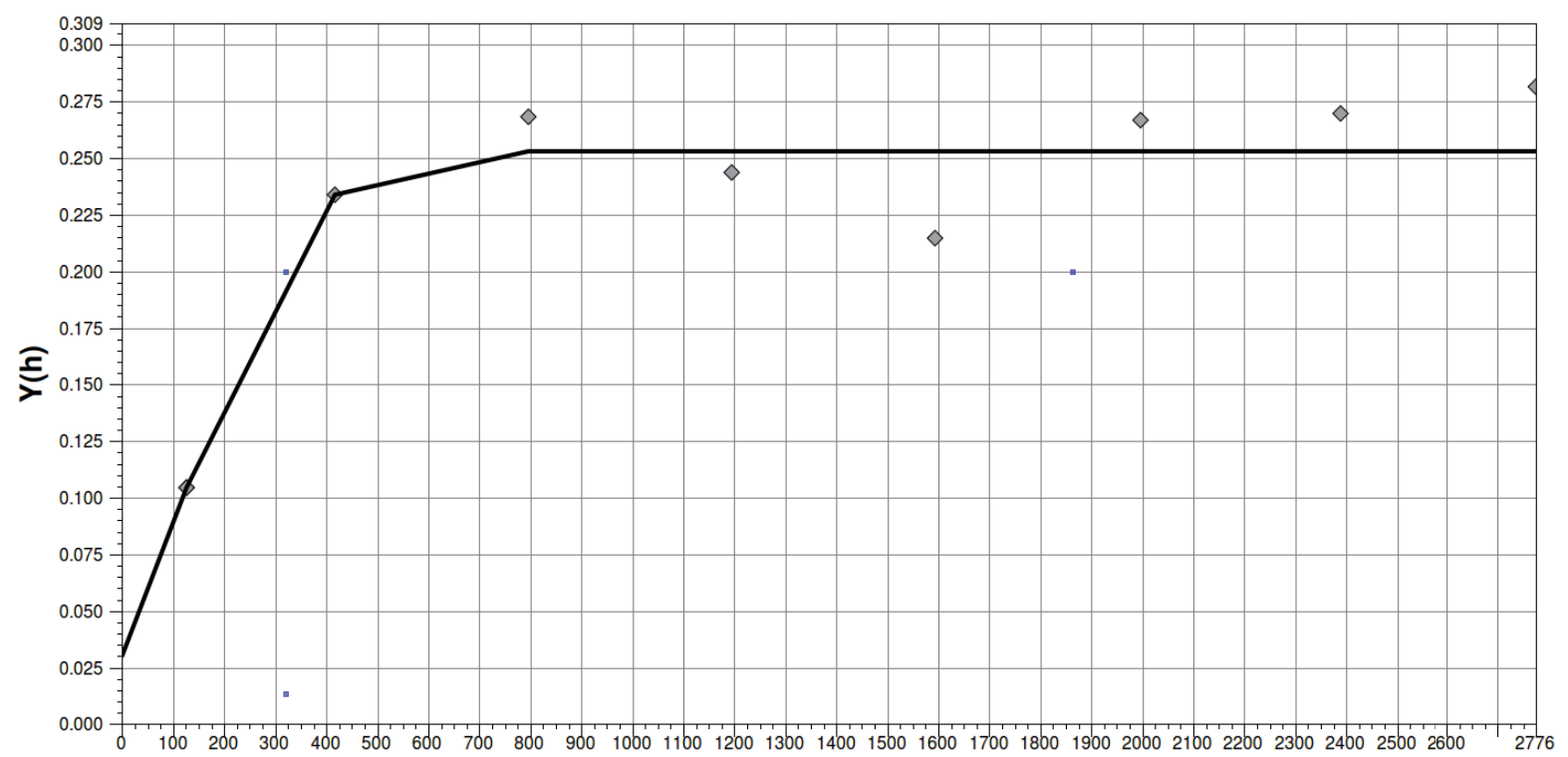

Distância

Figura 4. Ajuste de semivariograma para a mediana.

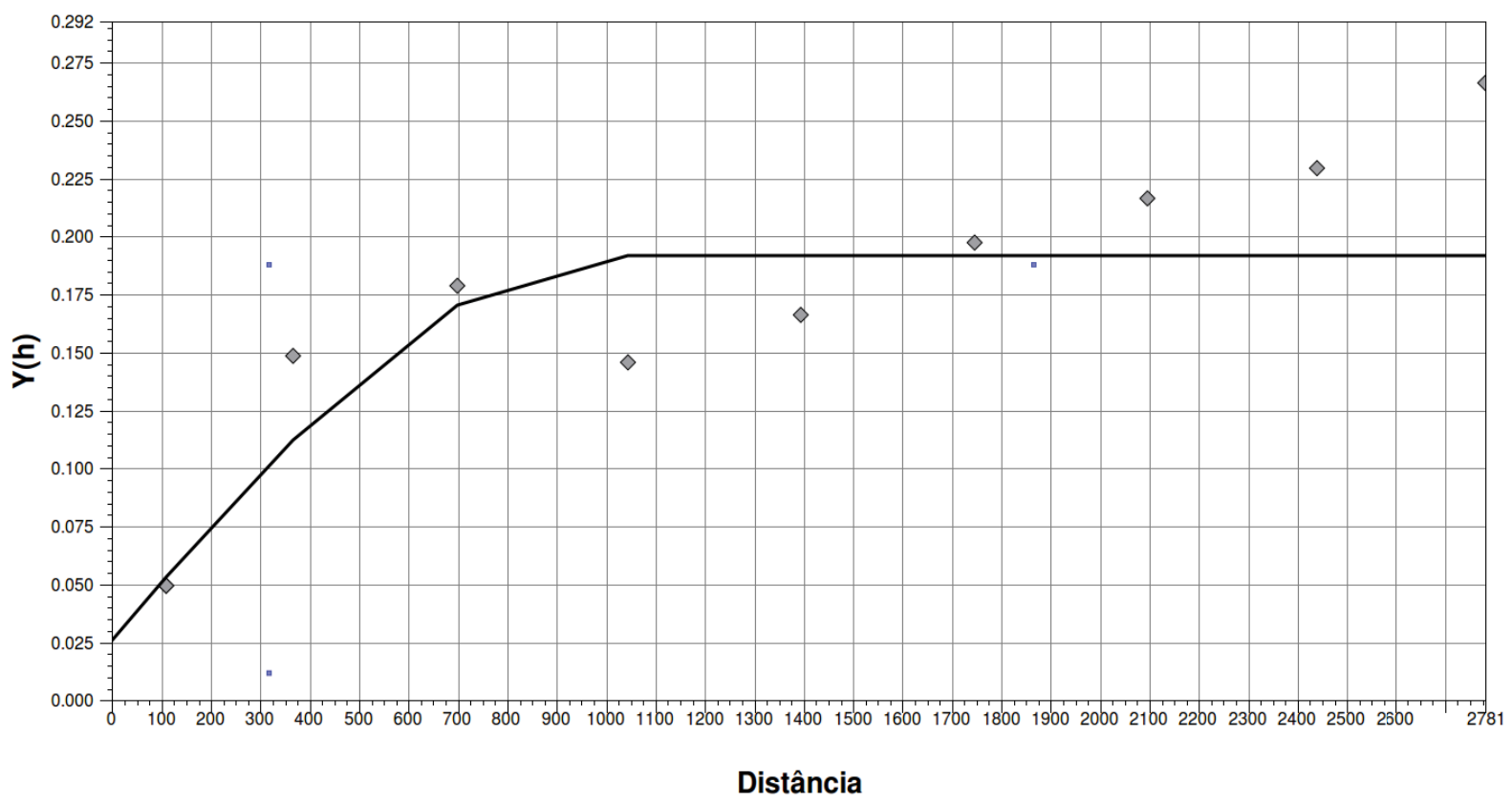

Figura 5. Ajuste de semivariograma para o quartil superior.

Os resultados de contribuição, apresentaram valores elevados, nos três limiares de corte, o que evidenciam os resultados anteriomente discutidos, ou seja, denotam um baixo efeito pepita e elevado valor de alcance.

Considerando os parâmetros adotados para ajuste dos semivariogramas gerados, obtivemos um modelo de krigagem por indicação, a partir de um modelo numérico de batimetria oriundo de um processo de krigagem ordinária sobre a grade de amostragem obtida (Figura 6). 


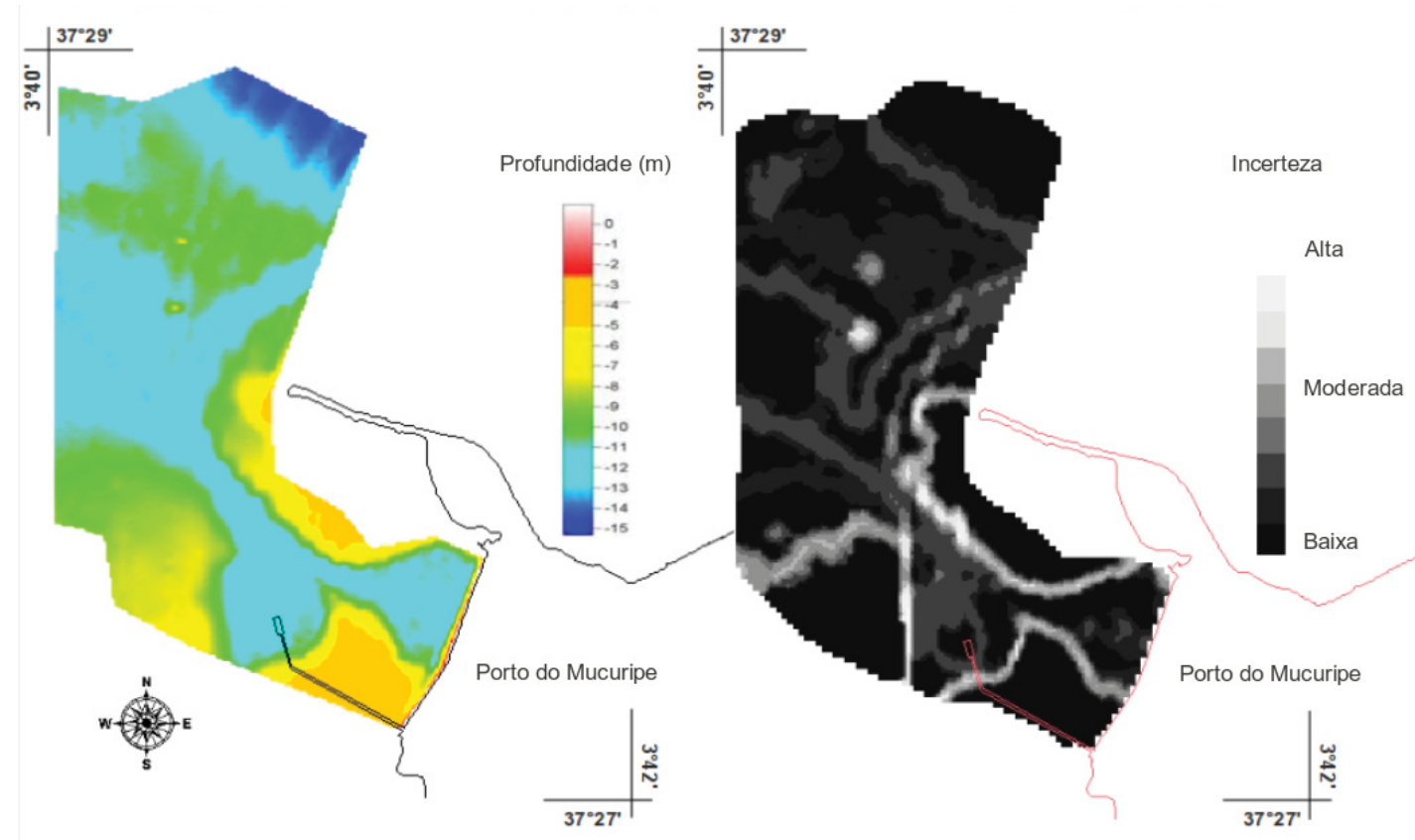

Figura 6 - A esquerda, têm-se o mapeamento obtido através do processo de krigagem ordinária, com a distribuição dos valores de profundidade para a região do canal de acesso do porto do Mucuripe. A direita, temos o modelo

obtido através de krigagem por indicação, identificando as regiões de maior incerteza do modelo ordinário.

Os resultados da figura 6, mostram que a conformação da topografia submarina apresenta o canal de navegação bem definido, com profundidade média em torno de -12 metros em toda sua extensão.

A análise por krigagem indicativa, mostra uma alta incerteza nas regiões que apresentam uma elevada diferença de profundidade. Esse resultado, é consistente com Løland e Høst (2005). Segundo os autores, em regiões com baixo grau de similaridade, os interpoladores geoestatísticos convencionais, apresentam dificuldade em detalhar com precisão os limiares de regiões com alta variabilidade batimétrica.

Para a entrada do canal principal, pode se verificar que o nível de incerteza varia de baixo a moderado em diversas posições. De modo similar, pode-se evidenciar a mesma distribuição para a região do atracadouro do porto do Mucuripe. Desse modo, considerando o modelo ordinário para esta região, verifica-se uma preponderância de baixa incerteza no modelo de krigagem ordinária.

Processos de sedimentação e alterações na morfologia batimétrica são comuns em regiões portuárias, motivo pelo qual há necessidade de dragagens de manutenção (Ying et al., 2012). Nesse íntere, este estudo, mostra o quão pertinente é a adoção de técnicas de geoprocessamento como ferramenta para a gestão portuária, monitorando a integridade dos canais de acesso aos atracadouros e reduzindo eventuais riscos a navegação costeira. Os resultados demonstram a viabilidade e conveniência de implementação de técnicas de krigagem por indicação em apoio aos levantamentos batimétricos, permitindo a análise da incerteza decorrente da interpolação comum.

\section{CONSIDERAÇÕES FINAIS}

Os resultados obtidos, demonstram o potencial e viabilidade da utilização de métodos de análise de incerteza geoestatística sobre dados batimétricos interpolados. Nesse âmbito, a adoção de métodos computacionais aplicados, representam uma importante recurso para otimização de custos e monitoramento de atividades de dragagem e manutenção de canais de acesso portuários.

A utilização do método de krigagem por indicação, possibilita verificar a confiabilidade de modelos previamente interpolados, considerando não somente a distribuição espacial dos pontos de 
amostragem, como também a variabilidade do atributo, permitindo inferir sobre possíveis incertezas em locais cuja malha amostral não se apresenta satisfatoriamente distribuída.

Para o modelo batimétrico obtido por krigagem ordinária, a partir de dados do levantamento realizado em 26 de Outubro de 2007 no canal de acesso do porto do Mucuripe - CE, é possível inferir que o modelo de referência apresentou em grande parte da região, níveis de incerteza classificados como baixos, com presença de regiões com moderada incerteza e picos de elevada incerteza sobre as regiões que apresentam ampla variação de profundidade.

\section{AGRADECIMENTOS}

Ao Instituto de Ciências do Mar - LABOMAR, pelo apoio logístico concedido para a elaboração deste trabalho.

\section{REFERÊNCIA BIBLIOGRÁFICA}

ALCÂNTARA, E. H. Análise da turbidez na planície de inundação de Curuai (PA, Brasil) integrando dados telemétricos de e imagens MODIS/TERRA. 217 p. (INPE-14621-TDI/1200). [Dissertação de Mestrado]. São José dos Campos (SP): Instituto Nacional de Pesquisas Espaciais, 2006.

BARBOSA, C.C.F. Sensoriamento remoto da dinâmica de circulação da água do sistema planície de Curuaí/ Rio Amazonas. 282 p. [Tese de Doutorado]. São José dos Campos (SP): Instituto Nacional de Pesquisas Espaciais, São José dos Campos, 2005.

CLARK, J. Coastal Zone Management Handbook. Nova York: Lewis Publisher/CRC Press, 1996. 720 p. DEUTSCH, C. V.; Journel, A. G. Geostatistical Software Library and user's guide. Nova York: Oxford University Press, 1998. 339 p.

Diretoria de Hidrografia e Navegação da Marinha do Brasil (2010). Tábuas de Marés. Acessado em 22 de maio de 2010 em: http://www.mar.mil.br/dhn/chm/tabuas/index.htm.

FELGUEIRAS, C. A. Modelagem ambiental com tratamento de incertezas em sistemas de informação geográfica: o paradigma geoestatístico por indicação. 215 p. (INPE-8180-TDI/760). [Tese de Doutorado]. São José dos Campos (SP): Instituto Nacional de Pesquisas Espaciais, 1999.

FILHO, H. A. G. Dragagem e gestão de sedimentos. 174p. [Dissertação de Mestrado]. Rio de Janeiro (RJ): Universidade Federal do Rio de Janeiro, 2004.

FLEMER, D.A.; RUTH, B. F.; BUNDRICK, C. M.; GASTON, G. R. Macrobenthic community colonization and community development in dredged material disposal habitats off coastal. Environmental Pollution. v. 96, n. 2, 1997. p. 141-154.

INTERNATIONAL MARITIME ORGANIZATION (2010). Acessado em 12 de fevereiro de $2010 \mathrm{em}$ : http://www.imo.org/home.asp

LI, J.; HEAP, A. D.; POTTER, A.; HUANG, Z. ; DANIELL, J.J. Can we improve the spatial predictions of seabed sediments? A case study of spatial interpolation of mud content across the southwest Australian margin. Continental Shelf Research. v. 31, 2011. p. 1365-1376.

LØLAND, A. ; HØST, G. Spatial covariance modelling in a complex coastal domain by multidimensional scaling. Environmetrics. v. 14, 2003. p. 307-321.

SECRETARIA ESPECIAL DE PORTOS (2010). Acessado em 12 de fevereiro de $2010 \mathrm{em}$ : http://www. portosdobrasil.gov.br/sistema-portuario-nacional.

VERFAILLIE, E.; LANCKER, V.; VAN MEIRVENNE, M. Multivariate geostatistics for the predictive modelling of the surficial sand distribution in shelf seas. Continental Shelf Research. v. 26, 2006. p. 2454-2468. YING, X. ; DING, P. ; WANG, Z.B. ; VAN MAREN, D.S. Morphological impact of the constrution of an offshore Yangshan deepwater harbor in the port of Shangai, China. Journal of Coastal Research. v. 1, 2006. p. 163-173. 